\title{
Estrategia metodológica para el fortalecimiento de habilidades sociales en los estudiantes de tercer grado del colegio "Juan Pablo II" de la ciudad de Potosí
}

\section{Methodological strategy for the strengthening of social skills in third grade students of the "Juan Pablo II" school in the city of Potosí}

\author{
Janneth Romano Choque \\ janne.rmn77@gmail.com \\ Universidad Autónoma Tomas Frías, Potosí - Bolivia
}

\section{RESUMEN}

El presente artículo hace referencia a las dificultades que presentan los estudiantes para relacionarse, es así que la educación se constituye en un espacio de convivencia importante donde los/as estudiantes se esfuerzan por establecer y mantener relaciones interpersonales, tratando de desarrollar identidades sociales y un sentido de pertenencia. Para su concreción se realizó un análisis teórico sobre, conceptos, componentes e indicadores de habilidades sociales, consultando a varios autores tarea que permitió tener una idea más amplia y clara sobre el tema y su relevancia en la educación. Se resume el diagnóstico, que se obtuvo a través de los instrumentos aplicados como: la encuesta dirigido a los estudiantes, la entrevista dirigida a los docentes y la guía de observación a estudiantes de secundaria tercero " $\mathrm{A}$ " del colegio "Juan Pablo II" de la ciudad de Potosí. El tipo de investigación que dio origen a este artículo fue descriptivo propositivo, lo cual permitió describir realidades observadas de forma natural tal y como se presenta la problemática investigada, identificando los rasgos característicos de la población donde se realizó la investigación, Se concluye que existe la necesidad de crear espacios de reflexión, para enfrentarse a los problemas con seguridad e independencia favoreciendo la hermosa tarea de educar.

Palabras clave: Estrategias metodológicas; adolescencia; habilidades sociales; relaciones interpersonales; espacios de convivencias

\section{ABSTRACT}

This article refers to the difficulties that students have in relating to each other, so that education is an important space for coexistence where students strive to establish and maintain interpersonal relationships, trying to develop social identities and meaning. of belonging. For its realization, a theoretical analysis was made on concepts, components and indicators of social skills, consulting several authors task that allowed to have a broader and clearer idea about the subject and its relevance in education. The diagnosis is summarized, which was obtained through the instruments applied such as: the survey aimed at students, the interview aimed at teachers and the observation guide for secondary students " $A$ " of the school "Juan Pablo II" of the city of Potosí. The type of research that gave rise to this article was descriptive purpose, which allowed describing realities observed naturally as the problem investigated, identifying the characteristic features of the population where the research was conducted, It is concluded that there is need to create spaces for reflection, to deal with problems with security and independence, favoring the beautiful task of educating.

Key words: Methodological strategies; adolescence; social skills; relationships; living spaces 
INTRODUCCIÓN

La experiencia educativa en los colegios, representa un espacio primordial donde no solo se deben generar conocimientos, pues en ella se dan muchas situaciones sociales de convivencia que encaminan al desarrollo integral y holístico de la persona; así como lo demanda la actual Ley 070, la cual se considera de vital importancia en el desarrollo de habilidades sociales, entendida como iniciativas y respuestas efectivas $\mathrm{y}$ apropiadas, las cuales influyen en el comportamiento verbal y no verbal, que no vienen determinadas de forma innata, al contrario son capacidades que se adquieren dando lugar a mejorar las relaciones sociales, ello es una búsqueda de convivencia positiva entre los estudiantes.

El deficiente uso de habilidades sociales entre los estudiantes de tercero " $A$ " del colegio "Juan Pablo II" de la ciudad de Potosí, se evidencia en conductas que muchas veces son difíciles de identificar y pasan como normales, como insultos, uso de sobrenombres, aislamiento, falta de valores como el respeto y empatía dando lugar a relaciones que se basan en el uso de una comunicación inapropiada, ya sea pasiva o agresiva, poca disposición para escuchar el punto de vista y necesidades del otro, relaciones en pequeños grupos, son factores que obstaculizan la convivencia sana $\mathrm{y}$ armónica entre pares.

En consecuencia, el presente artículo busca resaltar los hallazgos de la investigación los cuales responden a la necesidad de fortalecer las habilidades sociales, tomar en consideración el punto de vista ajeno, respetar su parecer aun cuando este sea distinto; manifestar opiniones sin temor a ser burlado o diferentes situaciones que se presenten. Su esencia radica en la habilidad para intercambiar mensajes, haciéndolo de forma honesta, respetuosa, directa y oportuna. Por lo tanto se diseña una estrategia metodológica de tal manera que se contribuya al fortalecimiento de habilidades sociales mismas que desarrollan conductas asertivas que generan la construcción de un ambiente y convivencia escolar armónico, a través de la promoción de relaciones de tolerancia, respeto, cordialidad y solidaridad entre ellos, donde además la persona pueda desenvolverse de manera autónoma y efectiva en la sociedad.

La escuela es un espacio de socialización en el cual se dan múltiples interacciones sociales y procesos de comunicación, donde los adolescentes deben enriquecer sus habilidades sociales, mediante la interacción con sus iguales y con los adultos.

Las habilidades sociales ofrecen una alternativa apropiada para enfrentar situaciones interpersonales conflictivas como: rechazar una petición, expresar desaprobación personal, ofrecer o recibir un halago, defender ideas, solicitar y exigir respeto de manera cordial, lo cual permitirá al estudiante ser responsable de sus propias acciones, cuidarse a sí mismo y relacionándose con los/as demás personas de manera positiva.

En los estudiantes de tercero "A" del colegio "Juan Pablo II", se observó un deficiente desarrollo de habilidades sociales entre los estudiantes, lo cual desfavorece la convivencia positiva, pues es evidente la existencia de barreras que dificultan el sano desenvolvimiento de las relaciones interpersonales, factores como la comunicación pasiva, timidez o miedo para expresarse, la falta de empatía, el no escuchar, puesto que tienden a interrumpir las conversaciones de los demás, represión de sentimientos de indignación o enfado, negación para trabajar en equipo, interacción en pequeños grupos que en grupo total, falta de valores como la responsabilidad, entre 
otros; impiden mantener la sensación de equilibrio que se anhela obtener en toda interacción social.

En este entendido se asevera, que estos factores han dado lugar a un clima hostil entre los estudiantes del tercero " $A$ " del colegio "Juan Pablo II" de la ciudad de Potosí, situación que debilita la convivencia sana entre pares, generando dificultades en las relaciones interpersonales.

Por lo antes descrito, se hace necesario que se genere un clima de agradable de convivencia, mejorando la forma de comunicarse y relacionarse con uno mismo y con los demás, que a su vez de lugar al apoyo emocional, ayuda, empatía, compañerismo y establecimiento de vínculos afectivos y de amistad.

\section{METODO}

El tipo de investigación que dio origen a este artículo fue descriptivo propositivo, lo cual permitió describir realidades observadas de forma natural tal y como se presenta la problemática investigada, identificando los rasgos característicos de la población donde se realizó la investigación, describiendo las causas y consecuencias para el existencia del problema sobre habilidades sociales.

Descriptivo porque permitió describir la realidad observada del estado actual de la problemática de investigación, identificando los rasgos característicos y particulares de los estudiantes de tercero "A" del colegio "Juan Pablo II" de la cuidad de Potosí. A su vez este tipo de investigación permitió conocer y analizar variables, teorías y enfoques, conceptos, dimensiones y componentes a partir de distintos autores que inciden en el desarrollo de habilidades sociales.

Propositivo por cuanto permitió generar conocimientos a partir del estudio y análisis de distintos factores y acontecimientos del problema de investigación para proponer una estrategia metodológica con métodos, técnicas, actividades, recursos y procesos que permiten el fortalecimiento de habilidades sociales en los estudiantes de tercero " $A$ " del colegio "Juan Pablo II" de la cuidad de Potosí.

La técnica de la observación contribuyó a la recogida de datos objetivos para lo cual se elaboró y procedió a su aplicación del instrumento: Guía de Observación no participante a los estudiantes de tercero "A" del colegio "Juan Pablo II" de la ciudad de Potosí.

Por su parte la técnica de la entrevista permitió al investigador formular preguntas a las personas capaces de aportarle datos de interés, estableciendo un diálogo peculiar, asimétrico, donde una de las partes busca recoger informaciones y la otra es la fuente de esas informaciones. Para ello el instrumento utilizado fue: Guía de entrevista dirigido a los docentes.

Por último, la técnica de la encuesta permitió recabar información de los estudiantes de tercero "A" del colegio "Juan Pablo II", aplicando el instrumento del cuestionario dirigido a estudiantes, elaborado mediante preguntas relacionados con los indicadores sobre las habilidades sociales en adolescentes.

En cuanto a la población de investigación estuvo constituida por: 450 estudiantes, 40 maestros, y una directora de Educación Secundaria comunitaria productiva de la Unidad Educativa "Juan Pablo II" de la ciudad de Potosí.

De la población total la muestra, utilizada fueron los estudiantes del tercero. " $A$ " del colegio "Juan Pablo II" de la ciudad de Potosí, el cual está constituido por 24 estudiantes de ambos sexos cuyas edades oscilan entre 13 a 15 años, asimismo 4 profesores para el desarrollo del diagnóstico, haciendo un total de 28 personas, ello porque se ha observado que en este grupo existe mayor índice de 
conductas agresivas y/o pasivas, poca tolerancia a los errores de sus compañeros quienes terminan siendo objeto de burla, poco interés hacia el proceso de enseñanza y aprendizaje lo cual da a entender que existe un deficiente desarrollo de habilidades sociales, por tanto el tipo de muestra es entendida como: no probabilística.

\section{Descripción del contexto de la investigación}

El trabajo de campo se desarrolló en el colegio "Juan Pablo II", ubicado en la Zona Villa Copacabana, entre la calle Pacífico Sequeiros y Laguna Colorada perteneciente al distrito municipal $\mathrm{N}^{\circ} 12$, en las faldas del cerro Ch'apini. El colegio alberga en sus ambientes a estudiantes que provienen de la misma zona, pertenecientes al distrito 12 , así mismo algunos estudiantes provienen de otros distritos cercanos a este. Señalan que al principio vivían solamente alrededor de 60 familias, en ese entonces quienes habitaban esa zona fueron inmigrantes paceños, los mismos que por la veneración a la virgen de Copacabana lo denominaron zona "Villa Copacabana" siendo su fundación en el año 1980.

El lugar donde se encuentra actualmente el colegio "Juan Pablo II" estaba ocupado por pastizales y promontorios de tierra, sin embargo, al transcurrir el tiempo la zona fue creciendo y se construyó el colegio, aunque con algunos conflictos a causa de los escasos recursos para la construcción.

El colegio "Juan Pablo II" (nivel secundario) fue fundada el 21 de septiembre de 1996, durante la presidencia de Jaime Paz Zamora, en sus inicios funcionaba al aire libre, desprovisto de todo material educativo $\mathrm{y}$ mobiliario necesario. Los directores de los distintos ciclos viendo la necesidad de facilitar a los estudiantes ambientes cómodos, buscaron distintas maneras de conseguir una infraestructura, dando a conocer la propuesta en diversas reuniones y congresos entre todo el plantel docente administrativo y los directores.

Luego de largos trámites lograron crear el Colegio "Juan Pablo II", gracias a la iniciativa y perseverancia de docentes, directores y padres de familia, finalmente el colegio abre sus puertas y es reconocida a nivel local y a nivel nacional, durante el mismo gobierno de Jaime Paz Zamora.

Ahora bien, las características sociales de los habitantes de la zona y la población estudiantil del colegió se relacionan a través del manejo de tres idiomas, en su mayoría son castellano hablantes producto de la influencia urbana, sin embargo, también manejan el quechua en un gran porcentaje y el aymara que es manejado por un reducido número de los estudiantes provenientes del departamento de La Paz.

En cuanto a las características económicas los estudiantes del colegio en su mayoría tienen padres que se dedican a diferentes actividades, como: comercio, choferes, albañiles, minería y agricultura. Asimismo, algunos padres de familia son técnicos superiores y otros se encuentran estudiando. Por lo tanto, el ingreso económico solo alcanza para el sustento del día de la canasta familiar, muchas de las familias provienen del área rural del departamento como: Tinquipaya, Cayara, La Palca, Yocalla, Porco, así como de otros departamentos del país Oruro, La Paz y Tarija, cabe destacar que pocas familias cuentan con viviendas propias, sino en calidad de alquiler.

Aunado a esto las características culturales de la población es variada y de diversas culturas, cabe señalar que los estudiantes del colegio son partícipes de la entrada folklórica cultural de "Ch'utillos" con la fraternidad Jalk'as, danza típica del municipio de Ravelo del departamento de 
Potosí; destacando así la riqueza cultural, alegría y devoción. Asimismo, realizan actividades que realzan aspectos culturales, donde realzan las danzas folklóricas del país y la región.

\section{RESULTADOS Y DISCUSIÓN}

\section{Análisis general del diagnóstico}

Tras la presentación de resultados de cada uno de los instrumentos aplicados a los estudiantes de tercero "A" del colegio "Juan Pablo II" de la ciudad de Potosí, se pudo evidenciar que las habilidades sociales entre los estudiantes es deficiente, por lo que se llegó a las siguientes conclusiones; con respecto a la dimensión conductual, se dedujo que existe un nivel bajo entre sus indicadores, pues los resultados muestran que la mayoría de los estudiantes no tienen desarrollado por completo la capacidad de escuchar, lo cual a su vez repercute en la facilidad para iniciar conversaciones, ya sea por temor o timidez, pues cuando uno se siente escuchado se siente valorado, lo que incide en el clima y cohesión entre pares, lo cual de acuerdo a los datos se encuentra en niveles bajos, puesto que mayormente interactúan en pequeños grupos.

En cuanto a la dimensión cognitiva, los porcentajes son preocupantes, debido a que los estudiantes solo a veces, pueden expresar sus pensamientos de manera justificada, debido a que la mayoría tiende a hablar en tercera persona y en plural, cosa que puede resultar negativa, si se hace sin consultar la opinión de los demás, del mismo modo no todos se sienten motivados a participar en actividades, si lo hacen es por obligación o por nota, lo que predispone a que muchos de los adolescentes sean fácilmente influenciados quizás porque hay una necesidad profunda de aceptación de los demás, lo que hace que muchas veces no se respete su postura.
Con respecto a la dimensión afectivoemocional, los datos se hallan entre medio $\mathrm{y}$ bajo, en una mayoría son las mujeres quienes expresan sus sentimientos de agrado como gratitud aunque ello sucede únicamente con adultos como: docentes, directora y otras personas; entre compañeros es muy raro que alguien haga un halago, en cuanto se refiere a la expresión de sentimientos de enfado o indignación la mayoría prefiere callar, asimismo entre compañeros no todos tienen la capacidad de comprender los sentimientos de los demás, comparten material ocasionalmente pero a cambio de algo, los estudiantes se rehúsan a trabajar en equipos formados por el docente, en ello influye la poca responsabilidad y compromiso con el equipo, poca relación amistosa y aceptación de todos, solo quieren trabajar entre amigos, en algunos casos prefieren trabajar individualmente.

\section{Estrategia metodológica para el fortalecimiento de habilidades sociales en los estudiantes de tercer grado del colegio "Juan Pablo II" de la ciudad de Potosí}

El diagnóstico expuesto, permite establecer las carencias respecto a las habilidades sociales y la necesidad de desarrollarlas; puesto que la relación entre los estudiantes, se basan en el uso de una comunicación inapropiada, ya sea pasiva o agresiva; poca disposición para escuchar el punto de vista y necesidades del otro, timidez para manifestar opiniones, divisionismo en pequeños grupos, dando paso a un clima hostil en el aula.

La estrategia metodológica contribuirá a fortalecer las habilidades sociales en los estudiantes de tercero "A" del colegio "Juan Pablo II" de la ciudad de Potosí, a partir de las necesidades señaladas, tomados en cuenta la 
operacionalización de la variable dependiente. Debido a que la escuela es un espacio de socialización en el cual se dan múltiples interacciones sociales y procesos de comunicación, donde los adolescentes deben enriquecer sus habilidades sociales, mediante la interacción con sus iguales, con los adultos y con autoridades.

Sin embargo, tradicionalmente la escuela ha considerado la interacción entre iguales como un fenómeno casi indeseable que alteraba el orden y la buena marcha de la clase, por ejemplo, la distribución espacial y del mobiliario en las aulas, que todavía hoy en día permanece en muchos contextos escolares, suele estar pensada más para evitar la comunicación entre los estudiantes que para favorecer su interacción. (Monjas y González Moreno, 2000).

Actualmente la Ley 070 considera no solo conocimientos, sino también espacios de reflexión, construcción y convivencia a través de las cuatro dimensiones: ser, saber, hacer y decidir, orientados al desarrollo integral y holístico de la persona. No obstante, al encontrarse aún en proceso de aplicación, todavía se mantiene la distribución espacial y del mobiliario en las aulas tradicionales.

En ese sentido, es importante comprender la importancia del desarrollo de habilidades sociales en el proceso de educación, porque que ello contribuye de manera significativa a la competencia personal, puesto que hoy en día el éxito personal y social parece estar más relacionado con la sociabilidad y las habilidades interpersonales de la persona que con sus habilidades cognitivas e intelectuales, lo cual no significa que no sean importantes (Monjas, 2002).

Asimismo la autora señala que la incompetencia social se relaciona con: baja aceptación, rechazo, ignorancia o aislamiento social por parte de los iguales, problemas escolares: bajos niveles de rendimiento, fracaso, absentismo, abandono del sistema escolar, expulsiones de la escuela, inadaptación escolar, problemas personales: baja autoestima, desajustes psicológicos: depresión, indefensión, inadaptación juvenil: delincuencia juvenil, alcoholismo, suicidio, toxicomanías.

Tener desarrollado habilidades sociales facilita y mejora las relaciones interpersonales, evitando el aislamiento, el rechazo social $\mathrm{y}$ tener una autoestima equilibrada. Disponer de habilidades sociales aporta múltiples beneficios, como: la libertad de decidir, la seguridad a la hora de enfrentarse a situaciones sociales, conocer y defender derechos así como la de los demás, entre otros. Todo esto influye de manera directa y positiva en las interacciones sociales como en la autoestima de la persona, generando espacios de convivencia armoniosa haciendo a la persona más estable emocionalmente y por ende más feliz.

\section{Fundamentos teóricos}

La propuesta desde una perspectiva filosófica se basa en lo comunitario, sustentado en el trabajo colaborativo, ello a través de la defensa de los derechos asertivos apreciados dentro de las habilidades sociales, considerado como un sistema de interacciones que organiza e induce la influencia recíproca entre los integrantes de un equipo, en el que cada miembro y todos se sienten mutuamente comprometidos con el aprendizaje de los demás, generando una interdependencia positiva que no implique competencia entre ellos.

En este sentido las habilidades sociales son importantes para el desarrollo del trabajo colaborativo donde la educación tiene el reto de preparar y formar integralmente a los estudiantes, de tal manera que estos posteriormente pueda desenvolverse sin 
mayores dificultades, poniendo en práctica las habilidades sociales, tales como: la capacidad de decir "no", la capacidad de pedir favores y hacer peticiones, la capacidad de expresar sentimientos positivos y negativos $y$ la capacidad de iniciar, mantener y terminar conversaciones.

Se asume como fundamento psicológico la experiencia educativa liberadora que fue asfixiada en el pasado y que hoy resurge en la nueva Ley 070 junto a las teorías desarrolladas por Vigotsky y Paulo Freire, cimentando así las bases de la nueva Educación Sociocomunitaria Productiva. Desde esta perspectiva es importante el desarrollo de una conciencia crítica y de actitudes reflexivas, a partir de la práctica de valores esenciales para la convivencia como la empatía, la honestidad, la libertad, la responsabilidad, el respeto, entre otros.

La propuesta también se fundamenta en el nuevo modelo sociocomunitario productivo, que promueve la formación integral, tomando en cuenta el aprendizaje comunitario, donde el elemento primordial es el dialogo, siendo el aprendizaje mutuo, entre docentes y estudiantes. El estudiante ya no es una hoja en blanco ni el profesor el que todo lo sabe, en este sentido la educación ya no es simple transmisión de conocimientos, pues se concibe la educación como, un acto de sociabilización y reflexión conjunta a través de un proceso de interacción democrática orientada hacia una convivencia armónica y sana, donde el desarrollo de habilidades sociales es muy importante.

Entendiendo que la educación está orientada a la formación integral de la persona debe dar lugar no solo a espacios para generar conocimientos, sino también espacios de convivencia que pongan como centro el vivir bien, encaminado hacia la formación holística de la persona, así como lo demanda la actual Ley 070. En este entendido la propuesta permite el desarrollo de habilidades sociales entendida como la capacidad de expresar pensamientos, sentimientos e ideas de manera respetuosa, en la búsqueda de una convivencia positiva y sana entre la comunidad educativa. 


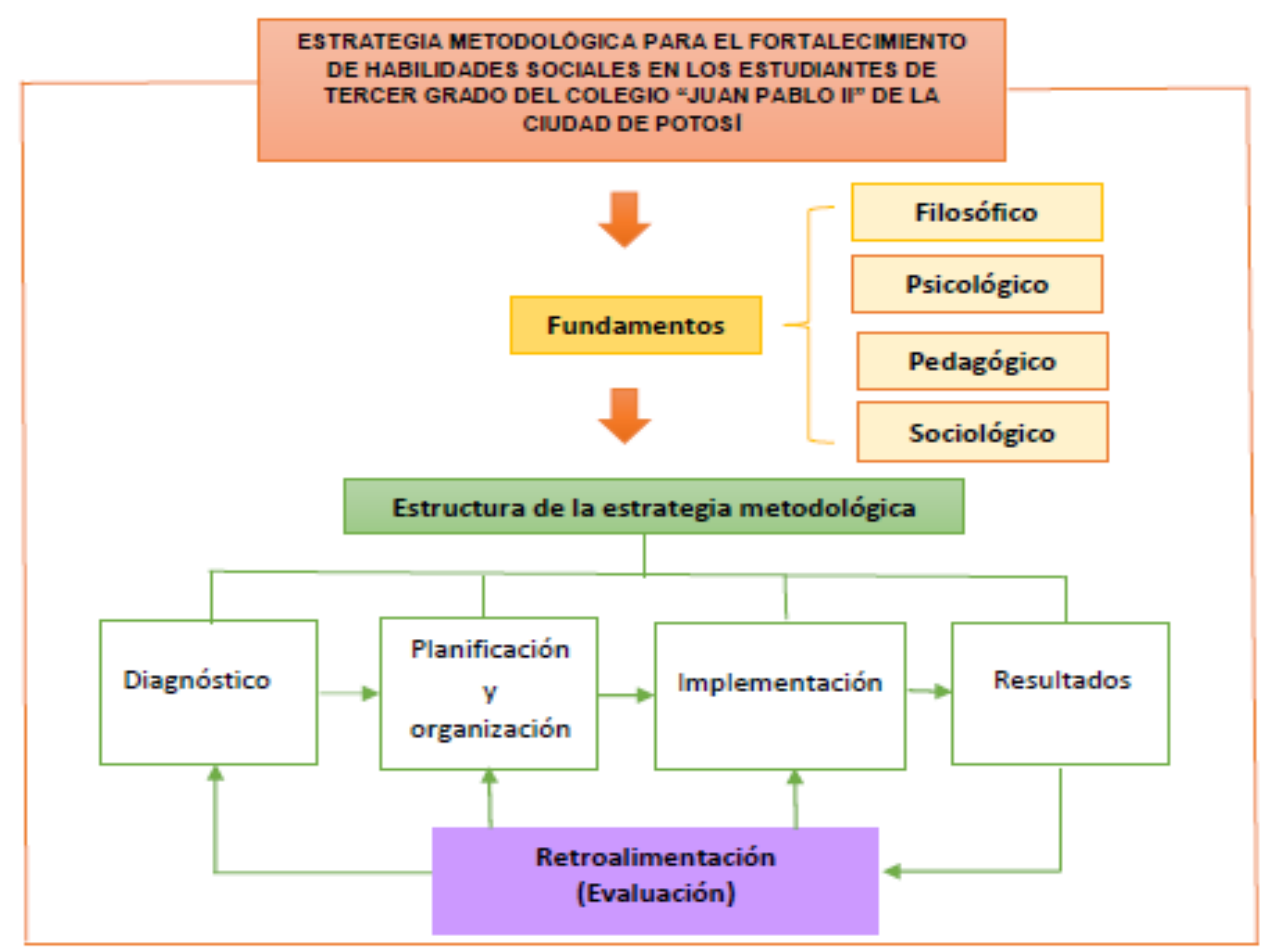

Figura 1. Esquema de la estrategia metodológica

\section{Estructura de la estrategia metodológica}

- Diagnóstico es la etapa preliminar el cual permitirá conocer el grado de conocimiento que los estudiantes tienen sobre el tema así como identificar el discernimiento sobre habilidades sociales.

- Planificación y organización a través de esta etapa que toma en cuenta distintos métodos y técnicas, se propicia la participación del grupo de estudiantes, el cual constituye una poderosa herramienta para formar integralmente a los estudiantes.

- Implementación es la etapa más importante, porque se desarrolla las diferentes actividades orientados por las temáticas organizadas en función a la operacionalización de la variable dependiente para lo cual se debe proveer los medios que se utilizaran.
- Resultados se espera conseguir que los estudiantes tengan mayor desenvolvimiento para expresarse en su entorno social de manera asertiva.

- Retroalimentación esta etapa cierra el ciclo del procedimiento de la implementación de la estrategia metodológica, pero no con un carácter terminal del proceso, sino todo lo contrario, servirá como una herramienta que permitirá la expresión de opiniones, juicios fundados, con los aciertos y errores, fortalezas y debilidades para así lograr la evaluación de la propuesta.

\section{Discusión}

A lo largo de los años se dio una evolución importante de diferentes términos hasta llegar a las habilidades sociales. Primero Salter empleó la expresión "personalidad excitatoria", que más tarde Wolpe sustituyó por la "conducta asertiva". 
Posteriormente, algunos autores propusieron cambiar el término por otros nuevos, "libertad emocional" por Lazarus en 1971, "efectividad personal" por Libernan y Cols en 1975, entre otros en los setenta del siglo XX, se emplea el término de habilidades sociales debido a su mayor implantación, estos autores fueron citados por (Caballo, 2007).

Uno de los términos más difundidos es el de habilidades sociales del cual se atribuye su origen a Salter en 1949, uno de los padres de la terapia de la conducta quien en su libro Conditioned Reflex Therapy (Terapia de reflejos condicionados), basado en la necesidad de aumentar la expresividad de los individuos describió seis técnicas como: la expresión verbal y facial de las emociones, el empleo deliberado de la primera persona al hablar, el estar de acuerdo cuando se reciben alabanzas, el expresar desacuerdo y la improvisación finalmente la actuación espontánea. (Choque Larrauri \& Chirinos Cáseres, 2009).

Más tarde el trabajo de Salter fue continuado por Joseph Wolpe en 1958, quien introduce por primera vez el concepto de asertividad, término que luego llegaría a ser sinónimo de habilidad social. Dicho autor utiliza el término asertivo como la expresión adecuada dirigida a otras personas, de cualquier emoción, Wolpe se enfocó en las expresiones de molestia y enfado. Posteriormente fue desarrollada por Arnold Lázarus en 1966, asimismo en el campo de las habilidades sociales han trabajado autores como Hersen, Bellack y Gil. (Caballo, 2007).

Otra fuente importante de las habilidades sociales son los trabajos efectuados por Zigles y Phillips en 1960,1961; dichos autores se refieren a las habilidades sociales como "competencia social". Sus trabajos con adultos hospitalizados sostienen que cuanto mayor es la competencia social previa de los pacientes que son internados en el hospital menor es la duración de su estancia en él y más baja su tasa de recaídas (Caballo, 2007, pág. 2).

Por otro lado, Goldstein en 1980 aporta con una clasificación bien estructurada y delimitada de las habilidades sociales, misma que consta de: primeras habilidades sociales, habilidades sociales avanzadas, habilidades relacionadas con los sentimientos, habilidades alternativas a la agresión, habilidades para hacer frente al estrés y habilidades de planificación. (Goldstein, Sprafkin, Gershaw, \& Klein, 1989).

Entre los métodos del desarrollo de habilidades sociales más influyentes, se destaca el Manual de evaluación y tratamiento de Habilidades Sociales de Caballo de 1986. En el texto, el autor hace un estudio minucioso del constructo y aporta con las dimensiones asertividad-pasividadagresividad como estilos de interacción comunes en las relaciones sociales. (Ramos, Cantillo, y Bestard, 2014).

Asimismo Choque Larrauri y Chirinos Cáceres (2009) señalan que los comienzos de la investigación con respecto a habilidades sociales provienen del campo de la terapia de la conducta y es a partir de los años 90 que recién se dirigió al campo educativo. Por lo cual a partir de ese año se le dio importancia al desarrollo de las habilidades sociales para la vida en los colegios por ser espacios ideales de aprendizaje.

Con relación a la adolescencia y las habilidades sociales es importante señalar que la adolescencia se conoce como un período posterior a la niñez que comienza con la pubertad. Su intervalo de duración varía según la fuente, pero generalmente enmarca su inicio entre los 10 y 12 años y su finalización a los 19 o 20. Esta etapa representa una época muy desafiante, porque las relaciones con los padres, los pares, y otras personas se hacen muy complejas, 
puesto que no son las de la infancia, pero que todavía no coinciden con las de los adultos, donde las relaciones sociales cobran gran relevancia, es así que las habilidades sociales se convierten en un elemento clave para alcanzar un buen ajuste y funcionamiento social en diferentes ámbitos de la vida.

En este sentido, Zavala, Valadez y Vargas (2008) exponen que, la adolescencia es un período crítico para la manifestación y el desarrollo de la inteligencia emocional y de las habilidades sociales. Por un lado, los adolescentes han dejado atrás las formas de socialización generalizada de la niñez para relacionarse con sus pares en la búsqueda de comprensión y aceptación social; el entendimiento de lo social se vuelve mucho más complejo, ya que en la adolescencia se abandona el acatamiento pasivo de las normas sociales en favor de un comportamiento más crítico y desafiante de las normas que enfrentan; por otra parte, tienen mayor presión por parte de padres y profesores ya que se espera de ellos comportamientos más elaborados.

En la misma línea Gutiérrez Carmona (2015) manifiesta que en este período, el individuo debe desenvolverse en múltiples tareas que implican relaciones interpersonales diferentes a las de la infancia y debe desarrollar habilidades para resolver problemas de manera independiente. Los adolescentes deben hacer amigos (as), aprender a conversar tanto con iguales, como con los adultos, deben participar en diferentes actividades al margen del círculo escolar y principalmente deben sentirse identificados e integrarse en un grupo.

En este entendido las habilidades sociales juegan un papel importante en la capacidad individual para encarar los desafíos de la edad adolescente ya que aportan beneficios importantes como es el caso de la aceptación social. La aceptación social es la condición personal de un individuo con respecto de un grupo de referencia; se relaciona con el liderazgo, la popularidad, el compañerismo, la jovialidad y el respeto". (Zavala Berbena, Valadez Sierra, y Vargas Vivero, 2008).

\section{CONCLUSIONES}

Los fundamentos que sustentan el marco teórico con relación a las habilidades sociales, respaldan el criterio que su desarrollo es una necesidad básica en las relaciones interpersonales de tal modo que generen la convivencia sana en la comunidad educativa que a su vez repercute de manera positiva en proceso de enseñanza aprendizaje.

Los resultados obtenidos del diagnóstico mediante la aplicación de instrumentos a los estudiantes y profesores, permitieron evidenciar que existe un deficiente desarrollo de habilidades sociales entre los estudiantes puesto que presentan dificultades para relacionarse como grupo con total inhibición para expresarse, lo cual es una barrera para la sociabilidad.

En este sentido, lo descrito anteriormente ha permitido la elaboración de la propuesta de una estrategia metodológica orientada a fortalecer habilidades sociales, conformada por cinco etapas de manera sistémica, cuyo desarrollo se aplica mediante una planificación curricular compuesta por objetivos, métodos participativos, técnicas, dinámicas, formas de organizar y distintos medios, encaminado al logro de objetivos propuestos.

Es importante seguir profundizando los fundamentos teóricos que se han considerado, se recomienda investigar aspectos relacionados con las habilidades sociales tales como comunicación, autoestima, $\mathrm{y}$ competencias comunicativas, puesto que estas teorías enriquecerán la formación integral del ser humano. 
Se debe profundizar el diagnóstico tomando en cuenta otros actores de la educación que influyen en el desarrollo de las habilidades sociales como: padres de familia y autoridades educativas, para determinar con mayor precisión qué otros factores implica el problema, lo cual dará lugar a una mejor comprensión del problema de investigación.

Para finalizar es necesario llevar a la práctica la propuesta sobre la estrategia metodológica mediante la ejecución y desarrollo de las actividades, planteados para fortalecer las habilidades sociales, misma que se enmarca en cinco etapas flexibles razón por la cual no solo puede ser aplicado en los estudiantes del colegio "Juan Pablo II" sino también puede ser implementada en otras instituciones interesadas en el tema de investigación de acuerdo a sus necesidades.

\section{REFERENCIAS}

Caballo, V. E. (2007). Manual de Evaluación y Entrenamiento de Las Habilidades Sociales. Madrid: Siglo XXI de España Editores S.A.

Choque Larrauri, R., y Chirinos Cáseres, L. J. (2009). Eficacia del Programa de habilidades para la vida en adolescentes escolares de Huancavelica Perú Revista de Salud pública., Volumen 11, 169-181

Goldstein, A. P., Sprafkin, R. P., Gershaw, N. J., y Klein, P. (1989). Habilidades sociales y autocontrol en la adolescencia: un programa de enseñanza. Barcelona: Martínez Roca

Gutiérrez Carmona, M. (2015). Inteligencia socio-emocional en la adolescencia: Diseño, implementación y evaluación de un programa formativo. Tesis doctoral. Granada

Monjas, I. (2002). Programa de enseñanza de habilidades de interacción social. (PEHIS). Madrid: CEPE

Monjas, I., y González Moreno, B. d. (2000). Las habilidades sociales en el currículo. Centro de Investigación y Documentación Educativa (C.I.D.E.).

Ramos, Y. C., Cantillo, A. F., y Bestard, C. M. (2014). Algunas consideraciones teóricas sobre las habilidades sociales. Revista de Información Científica., Recuperado de http://www.revinfcientifica.sld.cu/index. $\mathrm{php} / \mathrm{ric} / \mathrm{article} / \mathrm{view} / 1080 / 2482$

Zavala Berbena, M. A., Valadez Sierra, M. d., y Vargas Vivero, M. d. (2008). Inteligencia emocional y habilidades sociales en adolescentes con alta aceptación social. Revista Electrónica de Investigación Psicoeducativa, 6(2), Recuperado de https://www.redalyc.org/pdf/2931/293 121924004 